\title{
Approximation Theorems for Exponentially Bounded $\alpha$-Times Integrated Cosine Function
}

\author{
Lufeng Ling \\ School of Mathematics and Information Science, Shangqiu Teachers College, Shangqiu, Henan, China \\ Email: sqsxlfl@126.com
}

Received 26 September 2014; revised 25 October 2014; accepted 31 October 2014

Copyright (C) 2014 by author and Scientific Research Publishing Inc.

This work is licensed under the Creative Commons Attribution International License (CC BY). http://creativecommons.org/licenses/by/4.0/

c) (i) Open Access

\begin{abstract}
In this paper, based on the theories of $\alpha$-times Integrated Cosine Function, we discuss the approximation theorem for $\alpha$-times Integrated Cosine Function and conclude the approximation theorem of exponentially bounded $\alpha$-times Integrated Cosine Function by the approximation theorem of $n$-times integrated semigroups. If the semigroups are equicontinuous at each point $t \in[0, \infty]$, we give different methods to prove the theorem.
\end{abstract}

\section{Keywords}

$\alpha$-Times Integrated Cosine Function, Exponentially Bounded, Approximation

\section{Introduction}

Integrated semigroups were introduced by Arent [1] [2] and Davies and Pang [3] in 1987. The approximation theorem is one of the fundamental theorems in the theory of operater semigroups. There have been many results on approximation [4]-[7]. Cao [8] obtained the approximation theorem for $m$-times Integrated Cosine Function, $m \in N$. In this paper, we refine the theory by introducing $\alpha$-times Integrated Cosine Function for positive real numbers $\alpha$. Moreover, if the semigroups are equicontinuous at each point $t \in[0, \infty]$, we give different methods to prove the theorem.

Throughout this paper, we will denote by $X$-a Banach space with norm $\|\bullet\|$, by $B(X)$-the Banach space of all bounded linear operators from $X$ to $X ; A$ is a linear operator in $X$, by

$$
D(A), R(A), \rho(A), R(\lambda, A)
$$

respectively the domain, the range, the resolvent set, and the resolvent of $A$.

How to cite this paper: Ling, L.F. (2014) Approximation Theorems for Exponentially Bounded $\alpha$-Times Integrated Cosine Function. Advances in Pure Mathematics, 4, 580-584. http://dx.doi.org/10.4236/apm.2014.411066 


\section{Preliminaries}

Definition 2.1. Let $\alpha \in R^{+}$, then a strongly continuous family $\{S(t)\}_{t \geq 0}$ in $B(X)$ is called an $\alpha$-times Integrated Cosine Function, if the following hold:

1) $S(0)=0$;

2) For any $x \in X$, and $\forall s, t \geq 0$,

$$
\begin{aligned}
2 S(s) S(t)= & \frac{1}{\Gamma(\alpha)}\left\{(-1)^{\alpha} \int_{0}^{|t-s|}(|t-s|-r)^{\alpha-1} S(r) x \mathrm{~d} r\right. \\
& +\left(\int_{0}^{t+s}-\int_{0}^{t}-\int_{0}^{s}\right)(t+s-r)^{\alpha-1} S(r) x \mathrm{~d} r \\
& \left.+\int_{0}^{t}(t-s+r)^{\alpha-1} S(r) x \mathrm{~d} r+\int_{0}^{s}(t-s+r)^{\alpha-1} S(r) x \mathrm{~d} r\right\} .
\end{aligned}
$$

Definition 2.2. $A$ is a linear operator in $X, \alpha \in R^{+}, A$ is called the generator of an $\alpha$-times Integrated Cosine Function if there are nonnegative numbers $\omega, M$ and a mapping $S:[0, \infty) \rightarrow B(X)$ such that

1) $\{S(t)\}_{t \geq 0}$ is strongly continuous and $\left\|\int_{0}^{t} S(s) \mathrm{d} s\right\| \leq M \mathrm{e}^{w t}$ for all $t \geq 0$;

2) $(\omega, \infty)$ is contained in the resolvent set of $A$;

3) $R\left(\lambda^{2}, A\right)=\lambda^{\alpha-1} \int_{0}^{\infty} \mathrm{e}^{-\lambda t} S(t) \mathrm{d} t$ for $\lambda>\omega$.

Lemma 2.3. [9] For each $n \in N$ let $f_{n} \in L_{l o c}^{1}([0, \infty), X)$, with

$$
\left\|\int_{0}^{t} f_{n}(s) \mathrm{d} s\right\| \leq M \mathrm{e}^{\omega t}, \quad t \geq 0
$$

and let

$$
F_{n}(\lambda)=\int_{0}^{\infty} \mathrm{e}^{-\lambda t} f_{n}(t) \mathrm{d} t, \quad \lambda>\omega
$$

Assume that

$$
\lim _{n \rightarrow \infty} F_{n}(\lambda) \text { exists for } \lambda>\omega,
$$

and that for a fixed $t_{0} \in(0, \infty), \sup _{n \in N}\left\|f_{n}\left(t_{0}\right)\right\|<\infty$, and

$$
\lim _{h \rightarrow 0} \frac{1}{h} \int_{0}^{h}\left(f_{n}\left(t_{0}+s\right)-f_{n}\left(t_{0}\right)\right) \mathrm{d} s=0
$$

with uniform concergence for $n \in N$. Then $\lim _{m \rightarrow \infty} f_{n}\left(t_{0}\right)$ exists.

Lemma 2.4. [10] If $A$ is a linear operator in $X, \alpha \geq 0$. The following assertions are equivalent:

1) There exist constant $\omega, M \geq 0$, such that $\left(\omega^{2}, \infty\right) \subset \rho(A)$, and

$$
\left\|(\lambda-\omega)^{k+1}\left(\lambda^{1-\alpha} R\left(\lambda^{2}, A\right)\right)^{(k)}\right\| \leq M k ! .
$$

for $\lambda>\omega, \quad k \in N_{0}=N \bigcup\{0\}$.

2) $\forall \beta \in(\alpha, \alpha+1], A$ generate a $\beta$-times Integrated Cosine Function $\left\{S_{\beta}(t)\right\}_{t \geq 0}$, and exist constant $k$ such that $\alpha+1$-times Integrated Cosine Function $\left\{S_{\alpha+1}(t)\right\}_{t \geq 0}$ hold

$$
\limsup _{h \rightarrow 0} \frac{1}{h}\left\|S_{\alpha+1}(t+h)-S_{\alpha+1}(t)\right\| \leq k \mathrm{e}^{\omega t} \quad(t \geq 0, h \geq 0)
$$

\section{Main Results}

Theorem 3.1. If $A_{n}$ generates a $\alpha$-times Integrated Cosine Function $\left\{S_{n}(t)\right\}_{t \geq 0}$, and there is $M, \omega \in R^{+}$ 
such that $\left\|S_{n}(t)\right\| \leq M \mathrm{e}^{\omega t}$, then the following statements are equivalent:

1) $\lim _{n \rightarrow \infty} R\left(\lambda^{2}, A_{n}\right) x=R\left(\lambda^{2}, A_{0}\right) x, \forall x \in X$, for some $\lambda_{0}>\omega$, and $\left\{S_{n}(t)\right\}_{t \geq 0}$ is equicontinuous at each point $t \in[0, \infty]$;

2) $\lim _{n \rightarrow \infty} R\left(\lambda^{2}, A_{n}\right) x=R\left(\lambda^{2}, A_{0}\right) x, \quad \forall x \in X, \quad \lambda>\omega$, and $\left\{S_{n}(t)\right\}_{t \geq 0} \quad$ is equicontinuous at each point $t \in[0, \infty]$;

3) $\lim _{n \rightarrow \infty} S_{n}(t) x=S_{0}(t) x, \forall x \in X \quad$ uniformly on compacts of $t \geq 0$.

Proof: 1) $\Rightarrow$ 2) Consider the set

$$
\Omega=\left\{\lambda: \lim _{n \rightarrow \infty} R\left(\lambda^{2}, A_{n}\right) x=R\left(\lambda^{2}, A_{0}\right) x, \forall x \in X, \lambda>\omega\right\},
$$

which is nonempty by assumption.

Let $\mu \in \Omega$, then

$$
\lambda^{2}-A_{n}=\mu^{2}-A_{n}+\lambda^{2}-\mu^{2}=\left[I-\left(\mu^{2}-\lambda^{2}\right) R\left(\mu^{2}, A_{n}\right)\right]\left(\mu^{2}-A_{n}\right)
$$

when $\left|\mu^{2}-\lambda^{2}\right|<\frac{1}{\left\|R\left(\mu^{2}-A_{n}\right)\right\|}$

$$
R\left(\lambda^{2}, A_{n}\right)=R\left(\mu^{2}-A_{n}\right)\left[I-\left(\mu^{2}-\lambda^{2}\right) R\left(\mu^{2}, A_{n}\right)\right]^{-1}=\sum_{k=0}^{\infty}\left(\mu^{2}-\lambda^{2}\right)^{k} R\left(\mu^{2}, A_{n}\right)^{k+1}
$$

Obviously $R\left(\lambda^{2}, A_{n}\right)$ converges as $n \rightarrow \infty$. Therefore, the set $\Omega$ is open.

On the other hand, taking an accumulation point $\lambda$ of $\Omega$ with $\lambda>\omega$, we can find $\mu \in \Omega$, such that $\left|\mu^{2}-\lambda^{2}\right|<\frac{1}{\left\|R\left(\mu^{2}-A_{n}\right)\right\|}$. By the above considerations, $\lambda$ must belong to $\Omega$, i.e., $\Omega$ is relatively closed in $S=\{\lambda: \lambda>\omega\}$, which leads to the conclusion.

$$
\text { 2) } \Rightarrow 3 \text { ) Let } F_{n}(\lambda)=\lambda^{-\alpha+1} R\left(\lambda^{2}, A_{n}\right)=\int_{0}^{\infty} \mathrm{e}^{-\lambda t} S_{n}(t) \mathrm{d} t \text {, }
$$

for

$$
\begin{aligned}
& \lim _{n \rightarrow \infty} R\left(\lambda^{2}, A_{n}\right) x=R\left(\lambda^{2}, A_{0}\right) x \\
& \lim _{n \rightarrow \infty} S_{n}(t) x
\end{aligned},
$$

and $\left\{S_{n}(t)\right\}_{t \geq 0}$ is equicontinuous at each point $t \in[0, \infty]$; using Lemma 2.2, it is easy to know that $\lim _{n \rightarrow \infty} S_{n}(t) x$ exists. We now fix $b>0$, then for each $\varepsilon>0, \exists K \in N$; when $|t-s| \leq \frac{b}{K}, t, s \in[0, b]$, we have

$$
\left\|S_{m}(t)-S_{m}(s)\right\|<\frac{\varepsilon}{3}
$$

Pick $t_{i}=\frac{i}{k} b \in[0, b], i=1,2,3, \cdots, K$, then $\exists N_{0} \in N$ such that

$$
\left\|S_{n}\left(t_{i}\right)-S_{l}\left(t_{i}\right)\right\|<\frac{\varepsilon}{3}, n, l \geq N_{0}, i=1,2,3, \cdots, k .
$$

From (1) (2), we have $\left\|S_{n}(t)-S_{l}(t)\right\|<\frac{\varepsilon}{3}, \quad n, l \geq N_{0}, \quad t \in[0, b]$.

It shows that 3 ) is right.

3) $\Rightarrow 2)$ fix $t_{0} \in[0, \infty)$, for each $\varepsilon>0, \exists N_{0} \in N$, when $n \geq N_{0}$. 
We have

$$
\left\|S_{n}(s)-S_{N_{0}}(s)\right\|<\frac{\varepsilon}{3}, \quad s \in[0, t+1] .
$$

For $S_{n}(t)$ is continuous on $[0, t+1]$, then $\exists \delta_{0}>0,|s-t|<\delta_{0}$, when $s \in[0, t+1]$ We have

$$
\left\|S_{n}(s)-S_{n}(t)\right\|<\frac{\varepsilon}{3}, \quad n=1,2,3, \cdots, N_{0}
$$

Therefore, if $n \geq N_{0}, \quad s \in[0, t+1]$, then

$$
\left\|S_{n}(s)-S_{n}(t)\right\| \leq\left\|S_{n}(s)-S_{N_{0}}(s)\right\|+\left\|S_{N_{0}}(s)-S_{N_{0}}(t)\right\|+\left\|S_{N_{0}}(t)-S_{n}(t)\right\|<\varepsilon
$$

In conclusion $\left\{S_{n}(t), n \in N\right\}$ is equicontinuous at $t$.

By using the dominated convergence theorem, we obtain

$$
\lim _{n \rightarrow \infty} F_{n}(\lambda)=\lambda^{-\alpha+1} R\left(\lambda^{2}, A_{n}\right)=\int_{0}^{\infty} \mathrm{e}^{-\lambda t} S_{n}(t) \mathrm{d} t=\int_{0}^{\infty} \mathrm{e}^{-\lambda t} S_{0}(t) \mathrm{d} t
$$

So 2) is right.

$2) \Rightarrow 1$ ) the proof is obvious.

The proof is completed.

Corollary 3.2. If $A_{n}$ is the generator of $\alpha$-times Integrated Cosine Function $\left\{S_{n}(t)\right\}_{t \geq 0}$ satisfying:

$$
\left\|S_{n}(t+h)-S_{n}(t)\right\| \leq M \mathrm{e}^{\omega(t+h)} h^{\gamma}, n \in N, t, h \geq 0, \gamma \in(0,1]
$$

Then (1)-(3) are equivalent:

1) $\lim _{n \rightarrow \infty} R\left(\lambda^{2}, A_{n}\right) x=R\left(\lambda^{2}, A_{0}\right) x, \quad \forall x \in X$, for some $\lambda_{0}>\omega$.

2) $\lim _{n \rightarrow \infty} R\left(\lambda^{2}, A_{n}\right) x=R\left(\lambda^{2}, A_{0}\right) x, \quad \forall x \in X, \quad \lambda>\omega$.

3) $\lim _{n \rightarrow \infty} S_{n}(t) x=S_{0}(t) x, \quad \forall x \in X$, uniformly on compacts of $t \geq 0$.

Theorem 3.3. If $A_{n}$ is the generator of $\alpha$-times Integrated Cosine Function $\left\{S_{n}(t)\right\}_{t \geq 0}$, and there is $M, \omega \in R^{+}$such that $\left\|S_{n}(t)\right\| \leq M \mathrm{e}^{\omega t}, \quad \forall x \in X, \quad \lambda>\omega, \quad\left\{S_{n}(t)\right\}_{t \geq 0}$ is equicontinuous at each point $t \in[0, \infty]$. $\lim _{n \rightarrow \infty} R\left(\lambda^{2}, A_{n}\right) x=R\left(\lambda^{2}\right) x$ exist, for some $\lambda_{0}>\omega$, $\operatorname{ker} R\left(\lambda_{0}^{2}\right)=\{0\}$, then there is a linear operator $A$-generator of $\alpha$-times Integrated Cosine Function $S(t)$, such that $\lim _{n \rightarrow \infty} S_{n}(t) x=S(t) x, \forall x \in X$, and uniformly on compacts of $t \geq 0$.

Proof: By $\lim _{n \rightarrow \infty} R\left(\lambda^{2}, A_{n}\right) x=R\left(\lambda^{2}\right) x$, from the resolvent identity, we have

$$
R\left(\lambda^{2}, A_{n}\right)-R\left(\mu^{2}, A_{n}\right)=\left(\mu^{2}-\lambda^{2}\right) R\left(\lambda^{2}, A_{n}\right) R\left(\mu^{2}, A_{n}\right)
$$

then $R\left(\lambda^{2}\right)-R\left(\mu^{2}\right)=\left(\mu^{2}-\lambda^{2}\right) R\left(\lambda^{2}\right) R\left(\mu^{2}\right), \quad \lambda, \mu>\omega$ hence $\operatorname{ker} R\left(\lambda^{2}\right)$ and $\operatorname{Rang} R\left(\lambda^{2}\right)$ independent $\lambda$. Since $\operatorname{ker} R\left(\lambda_{0}^{2}\right)=\{0\}$, then there is a linear operator $A, D(A)=\operatorname{Rang} R\left(\lambda^{2}\right), R\left(\lambda^{2}\right) x=\left(\lambda^{2} I-A\right)^{-1} x$.

By Definition 2.2, we know that

$$
\lambda^{1-\alpha} R\left(\lambda^{2}, A_{n}\right) x=\int_{0}^{\infty} \mathrm{e}^{-\lambda t} S_{n}(t) x \mathrm{~d} t, \forall x \in X, \lambda>\omega,
$$

for $\lim _{n \rightarrow \infty} R\left(\lambda^{2}, A_{n}\right) x=R\left(\lambda^{2}\right) x$ exist, by the proof of the Theorem 3.1, we obtain that

$$
\lim _{n \rightarrow \infty} S_{n}(t) x=S(t) x \text { exist, }
$$


hence $\lambda^{1-\alpha} R\left(\lambda^{2}, A\right) x=\int_{0}^{\infty} \mathrm{e}^{-\lambda t} S(t) x \mathrm{~d} t, \quad \forall x \in X, \quad \lambda>\omega$

then $A$ generates a $\alpha$-times Integrated Cosine Function $\{S(t)\}_{t \geq 0}$, such that $\lim _{n \rightarrow \infty} S_{n}(t) x=S(t) x, \forall x \in X$, and uniformly on compacts of $t \geq 0$.

\section{References}

[1] Arendt, W. (1987) Vector-Valued Laplace Transforms and Cauchy Problems. Israel Journal of Mathematics, 59, 327352.

[2] Arent, W. and Kellermaan, H. (1989) Integrated Solutions of Volterra Integro-Differential Equations and Applications. Pitman Research Notes in Mathematics, 190, 21-51.

[3] Davies, E.B. and Pang, M.M.H. (1987) The Cauchy Problem and a Generalization of the Hill-Yosida Theorem. Proceedings of the London Mathematical Society, 55, 181-208. http://dx.doi.org/10.1112/plms/s3-55.1.181

[4] Zheng, Q. and Lei, Y.S. (1993) Exponentially Bounded C-Semigroup and Integrated Semigroup with Nondensely Defined Generators I: Approximation. Acta Mathematica Scientia, 13, 251-260.

[5] Lizama, C. (1994) On the Convergence and Approximation of Integrated Semigroups. Journal of Mathematical Analysis and Applications, 181, 89-103. http://dx.doi.org/10.1006/jmaa.1994.1007

[6] Shaw, S.-Y. and Liu, H. (2002) Convergence Rates of Regularized Approximation Processes. Journal of Approximation Theory, 115, 21-43. http://dx.doi.org/10.1006/jath.2001.3650

[7] Campiti, M. and Tacelli, C. (2008) Approximation Processes for Resolvent Operators. Calcolo, 45, 235-245. http://dx.doi.org/10.1007/s10092-008-0152-5

[8] Cao, D.-X., Song, X.-Q. and Zhang, X.-Z. (2007) The Approximations of m-Times Integrated Cosine Functions. Mathematics in Practice and Theory, 37, 164-167.

[9] Xiao, T.-J. and Liang, J. (2000) Approximation of Laplace Transforms and Integrated Semigroups. Journal of Functional Analysis, 172, 202-220.

[10] Zhang, J.Z. (1997) $\alpha$-Times Integrated Cosine Function. Acta Mathematica Scientia, 17, 33-38. 
Scientific Research Publishing (SCIRP) is one of the largest Open Access journal publishers. It is currently publishing more than 200 open access, online, peer-reviewed journals covering a wide range of academic disciplines. SCIRP serves the worldwide academic communities and contributes to the progress and application of science with its publication.

Other selected journals from SCIRP are listed as below. Submit your manuscript to us via either submit@scirp.org or Online Submission Portal.
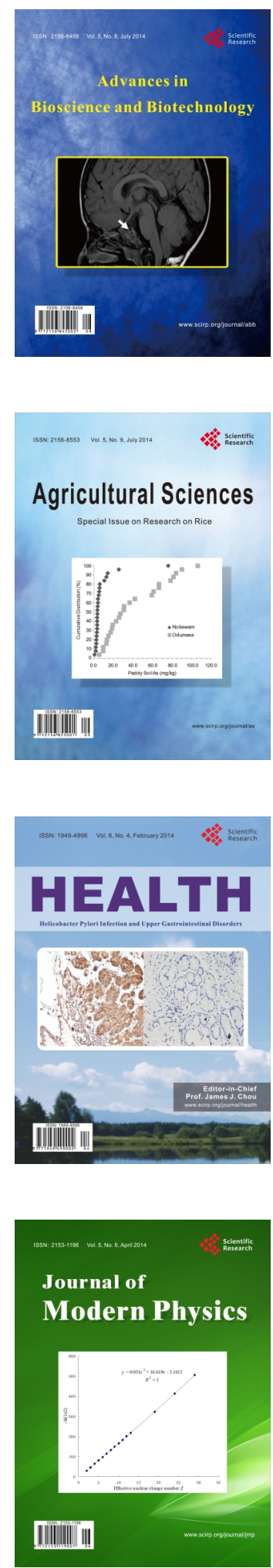
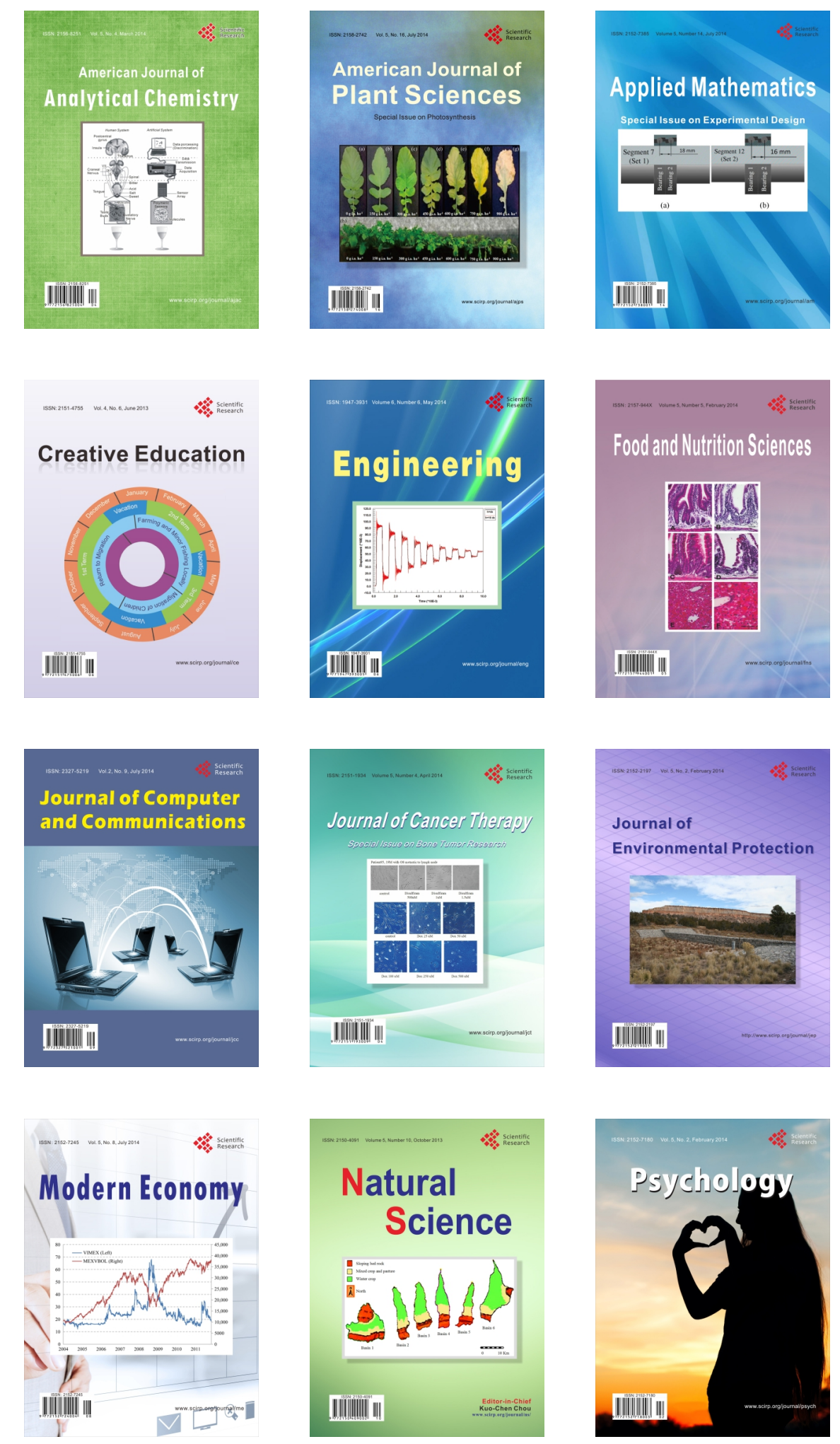